\title{
Resource Allocation for Scalable Video Broadcast in Wireless Cellular Networks
}

\author{
Junu Kim, Jinsung Cho, and Heonshik Shin
}

\begin{abstract}
Video broadcast services have become increasingly popular on packet-based wireless networks, such as 1xEV-DO and HSDPA which support high data rate. In this paper we propose a resource allocation algorithm for scalable video broadcast over such wireless networks. Our algorithm allocates time slots among the video layers of a scalable video and applies adaptive modulation and coding (AMC) to each video layer to maximize the sum of utilities for heterogeneous users with varying QoS requirements. It also considers competing video sessions and allocates time slots among them according to user preferences. Additionally, its polynomial time-complexity allows for online resource allocation that is necessary for real-time video services. Simulation experiments show that our algorithm outperforms a single-layer video broadcast with fixed modulation and coding (FMC), used in broadcast and multicast services (BCMCS) in the CDMA2000 system, and produce a near-optimal allocation.
\end{abstract}

Index Terms - resource allocation, scalable video broadcast, link adaptation, wireless cellular networks.

\section{INTRODUCTION}

$3^{\circ}$ G wireless networks such as 1xEV-DO [1] and HSDPA [2] provide users with a high data rate using packetbased transmission to support multimedia services, and this has motivated a lot of effort to bring video broadcast to these networks [4], [5], [7], [17]. The key challenge for video broadcasting is to allocate scarce wireless resources among receivers. This necessitates a better resource allocation scheme, which effectively improves the performance improvement of the wireless link.

In packet-based wireless networks, a channel resource consists of time slots. In a unicast service, link adaptation is useful to achieve a high data rate as the channel condition of a receiver often fluctuates. Changes of data rate are made by sending channel status information to the base station. The signal to interference and noise ratio (SINR) of the signal obtained by a receiver can vary over time by as much as $30-40 \mathrm{~dB}$, due to fast fading and its location in a particular cell. In order to improve the system capacity and peak data rate, $3 \mathrm{G}$ wireless networks such as 1xEV-DO or HSDPA hold the transmission power constant and use adaptive modulation and coding (AMC) as a method of link adaptation instead of power control. As shown in Fig. 1, a modulation scheme and a code rate are selected to maximize the data rate for for the

Manuscript received December 21, 2004.

Junu. Kim is with the School of Computer Science and Engineering, Seoul National University, Seoul 151-744, Korea (email: junukim@cslab.snu.ac.kr)

Jinsung. Cho is with the Dept. of Computer Engineering Kyung Hee University, Youngin 449-701, Korea (email: chojs@khu.ac.kr)

Heonshik. Shin is with the School of Computer Science and Engineering, Seoul National University, Seoul 151-744, Korea (email: shinhs@cslab.snu.ac.kr)

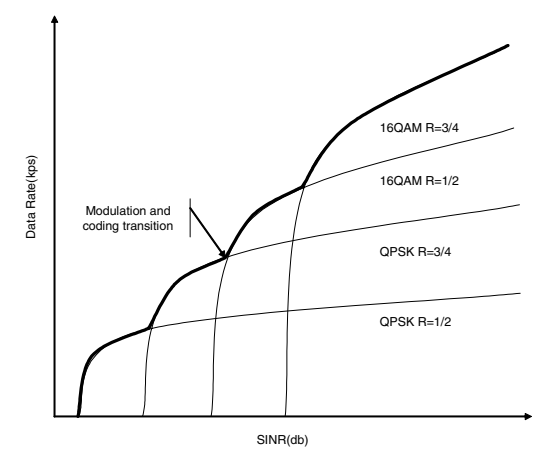

Fig. 1. Modulation and coding selection in adaptive modulation and coding.

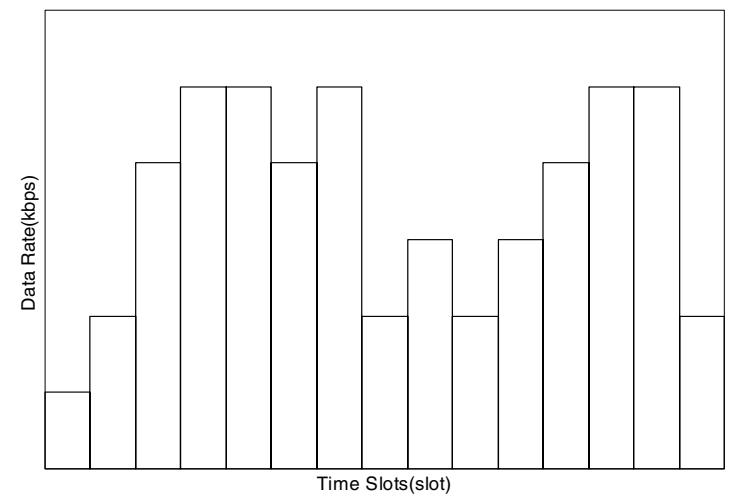

Fig. 2. Variable data rates of time slots in a packet-based wireless network.

SINR of the receiver. The base station in a cell schedules the data transmission of each receiver in the proper order and the data rate of each time slot in a packet-based wireless network varies over time, as shown in Fig. 2. To apply AMC in a broadcast environment, however, we need to consider several issues. In order to obtain acknowledgements from every receiver, we need unicast channels additional to those that already participate in the video broadcast. Moreover, we need to find out how to apply the aggregated channel status information to AMC in such an environment, which remains an open problem.

Recently the 3GPP-2 group laid the foundation of a specification for broadcast and multicast services (BCMCS) [4], [5] in a CDMA2000 network. This standard allows multiple receivers to share the same broadcast channels. It specifies 
that broadcast video streams are scheduled statically and that the resulting schedule is announced to receivers. Following this announcement, packets can be received without further communication. In order to maximize the number of receivers and to simplify the implementation, the standard forbids receivers from sending acknowledgements of received packets or reporting channel status. Therefore BCMCS do not use link adaptation but instead rely on fixed modulation and coding (FMC). With FMC, all time slots are transmitted with constant power, a single common modulation, and a coding scheme based on the SINR of all receivers is selected at the system level. The minimum acceptable SINR is predetermined from some appropriate consideration, and modulation/coding rates are selected to maximize the data rate.

We present a resource allocation algorithm which assigns resources among video sessions and the layers of each session while utilizing AMC to improve the performance in a broadcast environment where the receivers neither send acknowledgements nor report channel status. There are several issues in such a resource allocation. First, when we allocate resources to video layers in each session, we need to select a modulation/coding format for each layer which maximizes the aggregate qualities of the receivers requesting the session. Second, we need to assign resources between sessions. Because users prefer some video sessions to other sessions, we need to distribute resources according to user preferences: if users prefer one video to others, we need to allocate more resources to that session.

The remainder of this paper is organized as follows: Section II reviews some related work and Section III describes the problem formulation. In Section IV, we discuss the proposed algorithm and in Section $\mathrm{V}$ we evaluate the performance of the algorithm. Finally, concluding remarks are given in Section VI.

\section{RELATED WORK}

There has been a range of research activities on video streaming in both wired and wireless networks. In some approaches, the sender adapts the data rate of a video session for each receiver in an end-to-end fashion [7], [10]. In other systems, each video session is encoded in several layers and each receiver subscribes to a number of layers, depending on its network condition [17], [18]. This is effective in supporting receivers with heterogeneous data rates. In wireless networks, because the air resources are shared by all receivers, receiverdriven approaches can effectively utilize scarce air resources. A scalable video encoding such as MPEG-4 Fine-Granularity Scalable (FGS) [6] is widely used for receiver-driven approaches. A video stream is encoded in two classes of layer: the base layer and the enhanced layers. The enhanced layers are decoded in the context of the base layer and the quality of a video stream is progressively increased by receiving more layers. The receivers subscribe to the base layer and also to some of the enhanced layers, depending on their individual constraints and requirements.

There has been extensive work on layered video broadcast and multicast [7], [11]-[18]. McCanne et al. [7] proposed a receiver-driven algorithm for layered video multicast over a wired network. In their algorithm, each video layer is sent by means of separate multicast groups and each receiver adapts to changing channel conditions by periodically joining and leaving video layers. In other papers [11]-[13], [17], optimal algorithms for multicasting are presented. While some algorithms [11], [12] focus on the optimization of a single video session, Kar et al. [13] have suggested a global optimization algorithm. They mainly focus on wired networks, and thus ignore some characteristics of the wireless link.

Some bandwidth allocation algorithms for wireless networks have been proposed [14]-[16]. The algorithms adopt layered coding approaches in which the bandwidth of a video session is only allowed to take a set of discrete values, and the coding is adapted at the base station. The main purpose of this approach is to mitigate the possibility of an overload situation. Some work has focused on the performance improvement of video broadcast in wireless networks [17], [18]. Liu et al. [17] have proposed a generalized optimal wireless channel allocation algorithm with a general utility function for scalable video broadcast in a general wireless network. They have also considered user preferences and the layering overhead. Kang et al. [18] extended the existing broadcast and multicast services (BCMCS) standard in the CDMA2000 system by proposing an EDF-based scheduling technique that would adapt to receiver channel status. Their aim is to improve the performance of a practical broadcast standard. However, they do not utilize the characteristics of the underlying wireless links, but assume that each resource has the same property (i.e. fixed bandwidth or frequency).

We allocate resources among video layers in each video session, and also among video sessions, for scalable video broadcasts over wireless networks. The performance of the allocation process can be further improved by utilizing link adaptation in high-speed packet-based wireless networks such as $1 \mathrm{xEV}$-DO and HSDPA. In this paper we therefore develop a new resource allocation algorithm that includes link adaptation. The effect of the selected modulation and coding of each video layer on the data rate of a time slot is considered, and user preferences for some video session are also taken into consideration. The time complexity of our algorithm is designed to be low so that it can be used online.

\section{PROBLEM DESCRIPTION}

We now consider resource allocation in a single cell. A base station assigns wireless resources to receivers which are uniformly distributed within the cell. We assume that there is no control channel and that resources are fully used in video broadcasting. Because we are considering a packet-based wireless network such as 1xEV-DO and HSDPA, resources are represented by time slots. We assume that there are $N$ time slots in the system.

In AMC, the possible combinations of modulation schemes and the corresponding code rates in a wireless network are practically limited; therefore the possible data rates for a time 
TABLE I

PARAMETERS USED IN THIS PAPER.

\begin{tabular}{|c|c|}
\hline Parameter & Description \\
\hline$N$ & Total number of available time slots in the system. \\
\hline$S$ & Total number of video sessions in the system. \\
\hline$L$ & Total number of quality levels in the system. \\
\hline$M$ & Total number of receivers. \\
\hline $\begin{array}{c}M_{j, k} \\
B\end{array}$ & $\begin{array}{l}\text { The number of receivers which subscribe to the } k \text { th layer for the video } j \text {. } \\
\text { The data rate allocation unit in the system. }\end{array}$ \\
\hline$c_{k}$ & $\begin{array}{l}\text { The number of time slots for the } k \text { th layer in the system with the data rate } \\
\text { allocation unit } B \text {. }\end{array}$ \\
\hline$R$ & The data rate allocation matrix of the system. $R=\left(R_{1}, R_{2}, \ldots, R_{S}\right)$ \\
\hline$R_{j}$ & $\begin{array}{l}\text { The data rate allocation vector of the video } j . R_{j}=\left(r_{j, 1}, r_{j, 2}, \ldots, r_{j, L}\right) \text {, } \\
\text { where } r_{j, k} \text { is the number of data rate units allocated to layer } k \text { at video } j .\end{array}$ \\
\hline$r_{\min }$ & The minimum number of data rate units required for the base layer. \\
\hline$\mu_{j}\left(k, B \sum_{i=1}^{n} r_{j, i}, l_{j, k}\right)$ & $\begin{array}{l}\text { The utility function for a receiver of video } j \text {, where } k \text { is the requested quality } \\
\text { level of a receiver and } l_{j, k} \text { is the number of subscribed layers corresponding } \\
\text { to } j, k \text {. }\end{array}$ \\
\hline$\delta_{j}(k, n)$ & $\begin{array}{l}\text { The maximum utility of the receivers of video } j \text { which subscribes to the } k \text { th } \\
\text { layer under a given time slot budget of } n \text {. }\end{array}$ \\
\hline$h$ & The data rate overhead for layering. \\
\hline$U_{j}(k, n)$ & $\begin{array}{l}\text { The maximum session utility of video } j \text { after a } k \text { th layer is allocated under a } \\
\text { given slot budget of } n \text { time slots. }\end{array}$ \\
\hline$U\left(k_{j}, n\right)$ & $\begin{array}{l}\text { The maximum possible system utility after a } k \text { th layer is allocated to video } j \\
\text { under a given slot budget of } n \text { time slots. }\end{array}$ \\
\hline$U$ & The system utility. \\
\hline
\end{tabular}

slot have discrete values ${ }^{1}$. All possible data rates are indexed in ascending order, and we denote this index as the quality level. It is assumed that when a receiver subscribes to a video session, it also provides the desired quality level for that session, which is determined at the receiver based on channel status and the priority of the user. Among the possible quality levels that satisfy the desired packet error rate (PER) requirement of a receiver, the quality level which provides maximum data rate is selected; the receiver can also gain access to video layers with a lower quality level. When a receiver asks to subscribe to a video session, it sends a 'join' message and the desired quality level. After admission control at the base station, an 'accept' or 'reject' message is returned to the receiver. It is assumed that receivers do not send back any acknowledgement of received packets as would be the case in BCMCS.

The base station performs resource allocation according to the quality level requested by receivers. It allocates time slots among video sessions and among the layers of each session. Each video layer in a session is assigned a different quality level. The lowest quality level which is requested by receivers participating in the video session is assigned to the base layer. The remaining quality levels are assigned to the enhanced layers in increasing order. Sufficient time slots are always

\footnotetext{
${ }^{1}$ In HSDPA, the data rate of a time slot is determined by the modulation and coding selection (MCS) level. The selection of an MCS level is based on signal-to-interference-ratio (SIR) measured at a receiver. Under a given SIR, an MCS level which provides maximum throughput with is selected. In a CDMA system, the data rate option (DRC) level corresponds to the MCS level in HSDPA. There are 12 predefined modulation and coding combinations in the CDMA2000 system.
}

allocated to the base layer to accommodate all participating receiver that have passed admission control. The assignment process can be performed when a receiver joins or leaves to adapt to changes in the numbers of receivers.

Table I shows the parameters used in this paper. Time slots are allocated in a group, not separately, for the following reasons. First, the size of each video frame can be larger than a time slot, a frame can then be fragmented to several time slots. Thus grouping time slots can reduce the computation time with little quality degradation. Second, as the quality of a video is proportional to the data rate, allocating time slots in a fixed unit of data rate will greatly reduce evaluation time and algorithm complexity. The data rate allocation unit is denoted as $B$ and the number of time slots required to construct the data rate unit is denoted as $c_{k}$, where $k$ is the video layer with the $k$ th quality level to which the time slots are allocated. As the data rate at a higher quality level is greater than that of the lower quality level, for all $i, j$,

$$
\text { if } i<j \text { then } c_{i}>c_{j} \text {. }
$$

The assignment of data rates to video layers in a video session is represented by the resource allocation vector. The matrix of the vector in the system is 


$$
\begin{aligned}
R & =\left(\begin{array}{llll}
R_{1} & R_{2} & \cdots & R_{S}
\end{array}\right) \\
R_{j} & =\left(\begin{array}{c}
r_{j, 1} \\
r_{j, 2} \\
\cdots \\
r_{j, L}
\end{array}\right),
\end{aligned}
$$

where $R_{j}$ denotes the resource allocation vector for video session $j$, and $r_{j, k}$ is the number of data rate units assigned to the $k$ th layer. For a scalable video encoder [3], there is a practical limit to the minimum data rate of a base layer, which is denoted by $r_{\min }$. If layer 1 is the base layer, then $r_{j, 1} \geq r_{\min }$. As the allocation group size of the $k$ th video layer is $c_{k}$, the maximum value of $r_{j, k}$ is restricted by $\left\lceil\frac{N}{c_{k}}\right\rceil$ where $N$ represents the total number of time slots in the system. The constraints on $r_{j, k}$ are as follows: for $j=1, \ldots, S$,

$$
\begin{aligned}
r_{\text {min }} \leq r_{j, 1} \leq & \left\lceil\frac{N}{c_{1}}\right\rceil \\
0 \leq r_{j, k} \leq & \left\lceil\frac{N-S \cdot c_{1} \cdot r_{\text {min }}}{c_{k}}\right\rceil \text { for } k=2, \ldots, L \\
\text { where } & \sum_{i=1}^{L} c_{i} \sum_{j=1}^{S} r_{j, i} \leq N .
\end{aligned}
$$

To analyze the quality of the received video we need a utility function. The utility function of a receiver which subscribes to a video session $j$ at the $k$ th quality level is defined as follows:

$$
\mu_{j}\left(k, B \cdot \sum_{i=1}^{k} r_{j, i}, l_{j, k}\right)
$$

where $B \cdot \sum_{i=1}^{k} r_{j, i}$ is the cumulative data rate of a receiver which subscribes to the $k$ th video layer, and $l_{j, k}$ is the number of video layers to which time slots are allocated. Because the quality level requested by a receiver is the highest that it requires, a receiver subscribing to the $k$ th quality level can also have access to 1 st, 2 nd, ..., and $(k-1)$ th quality levels. Because we have assigned each quality level to a video layer, and the lowest quality level is assigned to the base layer, a receiver requesting the $k$ th quality level can access the 1 st, 2 nd, ..., and $k$ th video layers. Thus the total number of cumulative data rate units is $\sum_{i=1}^{k} r_{j, i}$, and the cumulative data rate of the receiver is $B \cdot \sum_{i=1}^{k} r_{j, i}$. The term $l_{j, k}$ is required because scalable encoding has some data rate overhead due to source coding or transport overhead. Since transport overhead increases according to the number of video layers, it is not negligible [8].

Although there is no consensus on utility functions for video streaming services, the well known peak-to-noise-ratio (PSNR) is widely used as a utility function [17]. PSNR in decibels $(\mathrm{dB})$ is computed as follows:

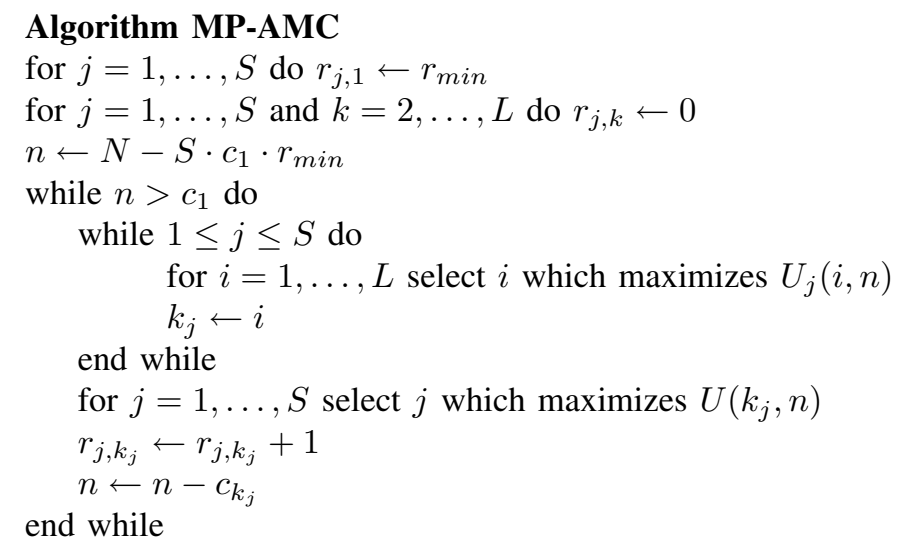

Fig. 3. Proposed algorithm: MP-AMC.

$$
\begin{aligned}
& P S N R=20 \log _{10}\left(\frac{255}{\sqrt{M S E}}\right) \\
& M S E=\frac{\sum(f(i, j)-F(i, j))^{2}}{N_{p}^{2}},
\end{aligned}
$$

where $f(i, j)$ is a source image which contains $N_{p} \times N_{p}$ pixels, and $F(i, j)$ is a reconstructed image decoded from the encoded source image. The PSNR of a video session can be calculated by averaging the PSNR of each frame in the video session.

The goal of our work is to maximize the system utility. The system utility is defined as the average of the utilities of all receivers, i.e.,

$$
U=\frac{1}{M} \sum_{j=1}^{S} \sum_{k=1}^{L} M_{j, k} \mu_{j}\left(k, B \sum_{i=1}^{k} r_{j, i}, l_{j, k}\right) .
$$

\section{Proposed Algorithm}

We propose an algorithm called MP-AMC which attempts to find the allocation matrix $R$ which maximizes the system utility. In order to be used online, the time complexity of the algorithm should be polynomial. To evaluate the performance of our algorithm, we consider two other algorithms: the optimal allocation algorithm with AMC (OPT-AMC) and the singlelayered video allocation algorithm with FMC (SINGLE-FMC). OPT-AMC is an exhaustive search algorithm which finds the optimal allocation matrix. SINGLE-FMC does exhaustive search for the optimal allocation when single-layered videos are used with FMC, as in BCMCS.

Our algorithm repeatedly picks up a video layer from all the layers of video sessions. The main idea of the selection method centers around the layer which maximizes possible system utility. The algorithm seeks the best possible allocation based on the quality level assigned to each video layer. The maximum possible system utility is the sum of the maximum possible utilities of all receivers. The utility of a receiver is maximized when all remaining time slots are assigned to the video layer corresponding to the quality level requested by the receiver. 
Fig. 3 shows the pseudocode for the MP-AMC algorithm. Initially, it distributes $c_{1} \cdot r_{\min }$ time slots to each base layer of all video sessions to meet the minimum data rate requirement for transmission at the lowest quality level. Next it iteratively selects the $k$ th layer of video session $j$, which maximizes the possible system utility under a given time slot budget of $n$. After a video layer has been chosen, the allocation vector $R_{j}$ and the number of available time slots are updated. Finally, the number of remaining time slots decreases by $c_{k}$.

$U_{j}(k, n)$ is the maximum possible session utility of session $j$ when a data rate unit is allocated to the $k$ th layer of the session under the given slot budget of $n$ time slots. It is the sum of the maximum possible utilities of the receivers which subscribe to the session. The maximum possible utility of the receivers which subscribe to the $k$ th video layer is denoted as $\delta_{j}(k, n)$. It is maximized when all available time slots are allocated to the $k$ th layer. If the number of available time slots is $n$, then the number of allocatable data rate units is $\left\lceil N / c_{k}\right\rceil$. Considering $\mu_{j}$ in Table $\mathrm{I}, \delta_{j}(k, n)$ can be defined as follows:

$$
\delta_{j}(k, n)=M_{j, k} \mu_{j}\left(k, B \cdot\left(\left\lceil\frac{n}{c_{k}}\right\rceil+\sum_{i=1}^{k} r_{j, i}\right), l_{j, k}\right) .
$$

$U_{j}(k, n)$ depends on the value of $k$ which is selected for the allocation of the data rate unit. Receivers which subscribe to layers lower than $k$ cannot utilize the allocation, and their number of available time slots is reduced by $c_{k}$. However, receivers which subscribe to the $k$ th layer can have $n$ time slots, because that data rate unit is assigned to the $k$ th layer. And the remaining receivers which subscribe to layers higher than the $k$ th layer can also utilize this allocation, although this reduces the number of available time slots by $c_{k} . U_{j}(k, n)$ is defined in Equation (7). The maximum system utility in allocating the $k$ th layer of video session $j$ is denoted as $U\left(k_{j}, n\right)$, and is defined as follows:

$$
U_{j}(k, n)= \begin{cases}\sum_{i=1}^{L} \delta_{j}\left(i, n-\left(c_{k}-c_{i}\right)\right) & \text { if } k=1 \\ \sum_{i=1}^{k-1} \delta_{j}\left(i, n-c_{k}\right) & \\ \quad+\sum_{i=k}^{L} \delta_{j}\left(i, n-\left(c_{k}-c_{i}\right)\right) & \text { if } 1<k \leq L .\end{cases}
$$

$$
\begin{aligned}
& U\left(k_{j}, n\right)= \\
& \frac{1}{M}\left(\sum_{i=1}^{L} U_{i}\left(0, n-c_{k_{j}}\right)-U_{j}\left(0, n-c_{k_{j}}\right)+U_{j}\left(k_{j}, n\right)\right) .
\end{aligned}
$$

Now we discuss the time complexity of our algorithm. The OPT-AMC algorithm searches all possible allocation matrices exhaustively to find the optimal solution. As shown in Inequality (4), $r_{j, i}$ satisfies $\sum_{i=1}^{L} c_{i} \sum_{j=1}^{S} r_{j, i} \leq N$. The time complexity of producing all possible allocations is greater than that of solving the inequality $c_{1} \sum_{i=1}^{L} \sum_{j=1}^{S} r_{j, i} \leq N$, because $c_{1}$ is greater than all other $c_{k}$, as shown in the statement (1). This problem is equivalent to producing all combinations of $N / c_{1}+L \cdot S-1$. As the total number of combination is $\left(\begin{array}{c}N / c_{1}+L \cdot S-1 \\ N / c_{1}\end{array}\right)$, OPT-AMC cannot run in polynomial time.

The MP-AMC algorithm can run in polynomial time. Although the number of iterations around the main loop varies, it is less than or equal to $\left\lceil N / c_{L}\right\rceil$ because $c_{L}$ is the smallest number of allocatable time slots at each iteration. The time complexity of the inner loop, which calculates $U_{j}(k, n)$, is $O\left(S \cdot L^{2} \cdot E\right)$, where $E$ is the time complexity for calculating the session utility. Therefore the time complexity of MPAMC is bounded by $O\left(\left\lceil N / c_{L}\right\rceil \cdot S \cdot L^{2} \cdot E\right)$, where $L$ and $N$ are constants for a given system. Thus resource allocation using MP-AMC can be done in polynomial time. In addition, because the data rate unit $B$ is fixed-size and the PSNR of a video is dependent on the data rate, we can calculate a possible PSNR for a video before the actual allocation. In that case the time-complexity of $O(E)$ can be substantially reduced.

\section{Performance Evaluation}

\section{A. Simulation Methodology}

We use the MoMuSys [3] reference MPEG-4 FGS codec as the encoder to generate bitstreams. As this is a bitplanebased scalable codec, the enhanced layer can be partitioned arbitrarily to make multiple enhanced layers. The lowest quality level is assigned to the base layer. For other quality levels the enhanced layer is partitioned into appropriate sizes. The minimum data rate required for the codec was obtained by testing: 36kbps. The video stream used in our simulation is the standard test stream: the Foreman (CIF) sequence. The layering overhead $h$ is set to $20 \%$.

To evaluate the proposed algorithm, a 1xEV-DO cellular network system is employed. We assume that the number of time slots in the system, $N$, is 600 because the length of a time slot is $1.667 \mathrm{~ms}$ in the $1 \mathrm{xEV}-\mathrm{DO}$ system and there are 600 time slots per second. We set the data rate allocation unit $B$ to $12 \mathrm{kbps}$, by considering the minimum data rate for the base layer and data rates of DRC steps. The quality level configuration is based on DRC steps, as defined in the 1xEVDO standard [1]. Each $c_{k}$ is calculated for the corresponding quality level. For example, we assign DRC step 12 to the $L$ th video layer and the data rate of a time slot which is allocated to the $L$ th layer is $(2457.6 \mathrm{kbps}) /(600 \mathrm{slots})=4.096 \mathrm{kbps}$. For a data rate unit of $12 \mathrm{kbps}, c_{L}=3$.

Suppose the number of receivers is 500 and they are uniformly distributed in a cell. A uniform distribution implies that the requested quality levels also generate a uniform distribution. The number of video sessions and the number of video layers are adjusted in each experiment.

\section{B. Experimental Results}

First, we evaluate the performance of MP-AMC when there is a single video session in the system, by varying the number of available time slots. Then we consider the case in which there are multiple video sessions and users prefer one video 


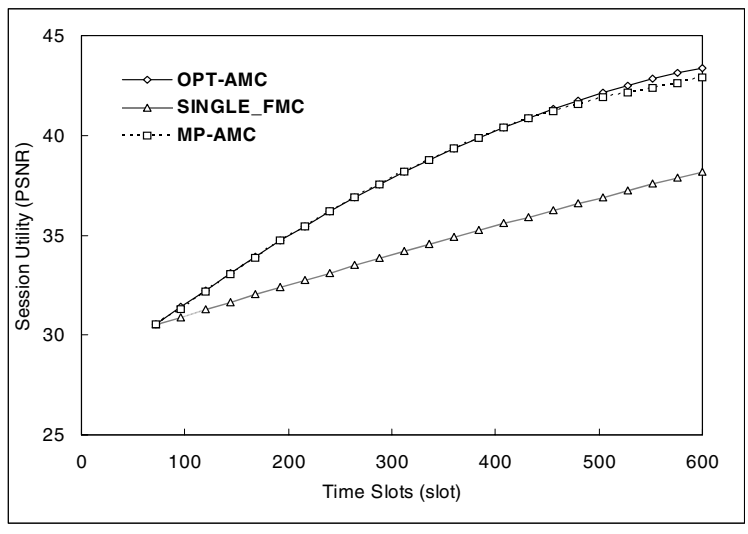

(a) Session utility

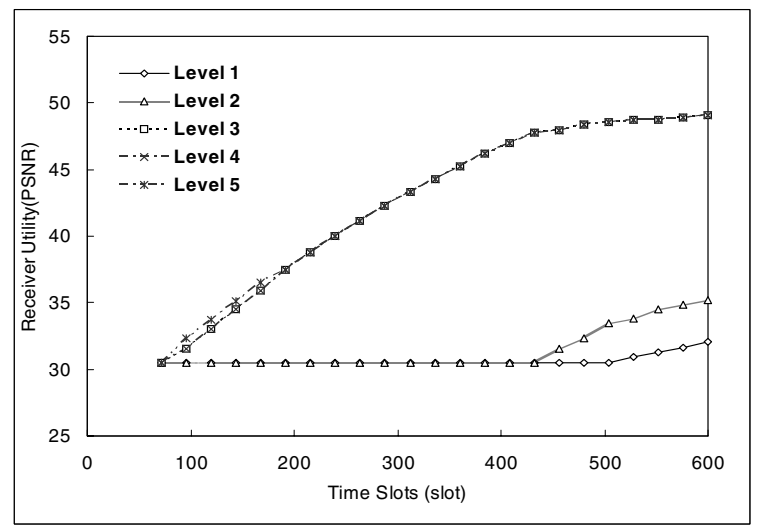

(b) Receiver utility with MP-AMC

Fig. 4. Session and receiver utility as a function of the number of time slots. $S=1, L=5$.

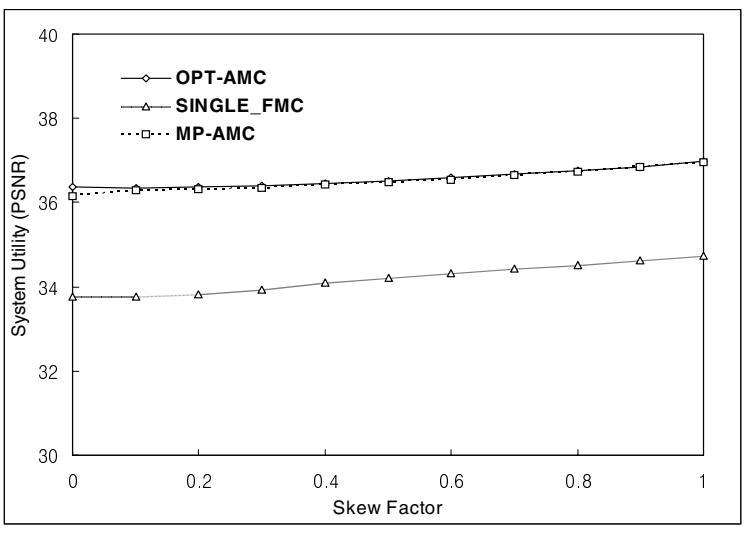

(a) System utility

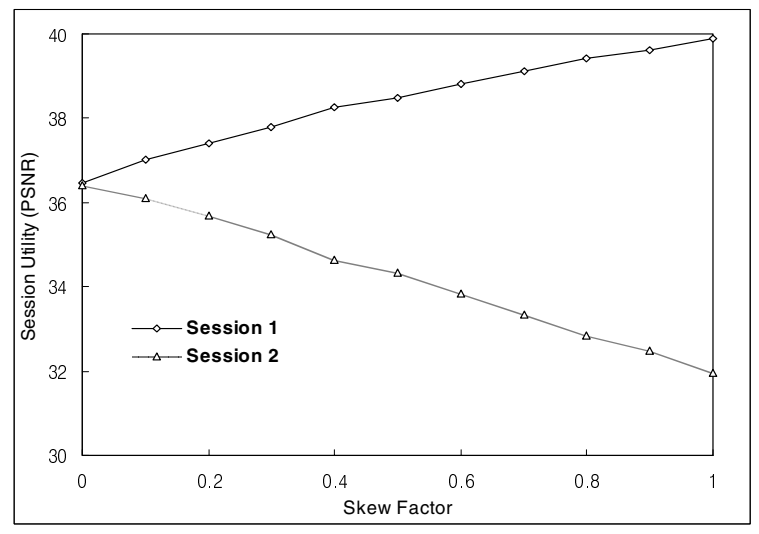

(b) Session utility with MP-AMC

Fig. 5. System utility as a function of the skew factor in the Zipf distribution. $S=2, L=4, N=600$.

session to the others. The system performance is expressed in terms of utility. The receiver utility is the PSNR value perceived at a receiver which subscribes to a session with a particular quality level and receives all video layers less than and equal to that level. The session utility is the average utility of all receivers subscribing to that session; and the system utility is the average utility of all receivers in the system.

Fig. 4 shows the allocation of time slots in a single-session environment. The number of video layers is 5 and the number of time slots available in the system is 600. In Fig. 4(a), the average difference between OPT-AMC and MP-AMC is $0.3 \%$, and does not exceed $1.2 \%$. The difference between OPT-AMC and SINGLE-FMC is $9.5 \%$ and, as the number of time slots in the system increases, the difference between OPT-AMC and SINGLE-FMC keeps increasing. For $N=600$, the difference approaches $12 \%$. Fig. 4(b) shows the utility of each receiver which subscribes to a certain quality level. Initially, additional time slots are assigned to high quality levels, and only later to lower quality levels. That is because the data rate of a time slot in one of the higher layer is greater than that in one of the lower layers, and the PSNR is proportional to data rate. Receivers that subscribe to a higher quality level benefit from the extra time slots and therefore the system utility increases.
We now consider the performance of the proposed algorithm in the multiple-session case, as well as the preferences of users for different video sessions. Generally, preferences can be modelled as a Zipf distribution [17] which is denoted as follows:

$$
p_{j}=\frac{\left(\frac{1}{j}\right)^{\theta}}{\sum_{j=1}^{S}\left(\frac{1}{j}\right)^{\theta}} \text { for } j=1,2, \ldots, S,
$$

where $\theta$ is the skew factor. When $\theta=0$, the Zipf distribution becomes the uniform distribution with $p_{j}=\frac{1}{S}$ for all value of $j$. As $\theta$ increases the probabilities are skewed. We can model the preferences of users for particular video sessions by increasing $\theta$.

Fig. 5 shows the system utility as a function of the skew factor when there are two video sessions. The average difference between the performance of OPT-AMC and MP-AMC is only $0.2 \%$ in Fig. 5(a). MP-AMC and SINGLE-FMC are separated by more than $2 \mathrm{db}$. MP-AMC outperforms SINGLEFMC right across the skew factor spectrum. Fig. 5(b) shows the utility of each session. When $\theta=0$, two sessions have the same utility. As the skew factor increases, the difference between the sessions increases and, when $\theta=1$, almost all 
the time slots are allocated to Session 1.

\section{CONCLUSION}

We have considered the practical problems of resource allocation for video broadcast in packet-based wireless networks. By utilizing AMC with scalable video broadcast, we can further improve the performance of the allocation process. The proposed algorithm MP-AMC is designed to allocate wireless resources among video layers and among video sessions simultaneously, and perform in polynomial time, making it's suitable for online use. It is simple but effective compared to the optimal allocation. Experiments have shown that our algorithm outperforms resource allocation based on singlelayer video with FMC. We have also demonstrated that our algorithm can adapt to a varying resource budget and to users' preferences for video sessions.

\section{REFERENCES}

[1] TIA/EIA/IS-856-1, cdma2000 High Rate Packet Data Air Interface Specification.

[2] H. Holma and A. Toskala, "WCDMA for UMTS," Radio Access for Third Generation Mobile Communications, 2nd Edition, John Wiley and Sons, 2002

[3] A. Pearmain, A. Carvalho, A. Hamosfakidis and J. Cosmas, "The MoMuSys MPEG-4 mobile multimedia terminal," Proc. 3rd ACTS Mobile Summit Conference, pp. 224-229, June 1998

[4] P. Agashe, R. Rezaiifar, P. Bender and QUALCOMM, "Cdma2000 high rate broadcast packet data air interface design," IEEE Communications Magazine, vol. 42, no. 2, pp. 83-89, February 2004.

[5] J. Wang, R. Shinnarajaj, T. Chen, Y. Wei, E. Tiedemann and QUALCOMM, "Broadcast and multicast services in cdma 2000," IEEE Communications Magazine, vol. 42, no. 2, pp. 76-82, February 2004.

[6] W. Li, "Overview of fine granularity scalability of MPEG-4 video standard," IEEE Transactions on Circuits and Systems for Video Technology, vol. 11, no. 3, pp. 301-317, March 2001.

[7] S. McCanne, V. Jacobson and M. Vitterli, "Receiver-driven layered multicast," Proc. ACM SIGCOMM'96, Stanford CA, August 1996.

[8] P. de Cuesto, D. Saparillam and K. W. Ross, "Adaptive streaming of stored video in a TCP-friendly context: Multiple versions or multiple layers," Proc. Int. Packet Video Workshop, Kyungju, Korea, April 2001.

[9] S. Chakrabati and R. Wang, "Adaptive control for packet video," Proc. of IEEE International Conference on Multimedia Computing and Systems, pp. 56-62, May 1994.

[10] N. Duffield, K. Ramakrishnan and A. Reibman, "SAVE: an algorithm for smoothed adaptive video over explicit rate networks," IEEE/ACM Transaction on Networking, vol. 6, no. 6, pp. 717-728, 1998.

[11] J. Liu, B. Li and Y. Q. Zhang, "An end-to-end adaptation protocol for layered multicast using optimal rate allocation," IEEE Trans. Multimedia, vol 6, pp. 87-102, Februrary 2004.

[12] Y. Yang, M. Kim and S. Lam, "Optimal partitioning of multicast receivers," Proc. IEEE ICNP'O0, November 2000.

[13] K. Kar, S. Sakar and L. Tassiulas, "Optimization based rate control for multirate multicast sessions," Proc. IEEE INFOCOM'01, Anchorage, April 2001.

[14] A. K. Talukdar, B. R. Badrinah and A. Acharya, "Rate adaptation schemes in networks with mobile hosts," Proc. of ACM/IEEE MobiCom'98, pp. 169-180, October 1998.

[15] S. K. Das, and S. K. Sen, "Quality-of-services degradation strategies in multimedia wireless networks,", Proc. of IEEE Vehicular Technology Conference, pp. 1884-1888, Ottawa, May, 1998.

[16] T. Kwon, Y. Choi and S. K. Das, "Bandwidth adaptation algorithms for adaptive services in mobile cellular network," Wireless Personal Communication Magazine, vol. 22, no. 3, pp. 337-357, 2002.

[17] J. Liu, B. Li, Y.-T. Hou and I. Chlamtac, "On optimal layering and bandwidth allocation for multi-session video broadcasting," IEEE Transactions on Wireless Communications, February 2004.

[18] K. Kang, J. Cho and H. Shin, "Dynamic packet scheduling for cdma2000 1xEV-DO broadcast/multicast services," IEEE WCNC2005, New Orleans, March, 2005 